\title{
Alignment Control of STEM: a Ronchigram Based Approach
}

\author{
S.W. van der Hoeven , A.J. den Dekker , A. Tejada
}

DCSC, Delft University of Technology, Mekelweg 2, 2628 CD, Delft, The Netherlands

Various advances have been made in the past towards automatic alignment of electron microscopes. Especially in the area of electron tomography automation is used $[1,2]$. In structural analysis and HR-STEM these auto-alignment procedures are combined with additional manual alignment to obtain the highest resolution images. This is often done using Ronchigrams, also called shadow images [3]. The research presented here concerns the development of a method to automatically align the microscope using Ronchigrams around Scherzer defocus. This as opposed to other research (e.g. [4,5]), where strong underfocus is used. To this end alignment information is extracted from the images. This information can then be used in a control program developed to adjust the microscope settings to optimize the alignment automatically.

The intensity in the Ronchigram image is [6]: $I_{R}(\mathbf{k})=(\mathbf{k}) \quad P(\mathbf{k})^{2}$ where $(\mathbf{k})$ is the object transmission function, $\mathbf{k}$ denotes the coordinates in reciprocal space, represents a convolution integral, and $P(\mathbf{k})$ is the probe function: $P(\mathbf{k})=A(\mathbf{k}) \exp (i(\mathbf{k}))$, where $A(\mathbf{k})$ is the aperture function and $(\mathbf{k})$ is the wave aberration function as described in for example [7]. Wave aberration information, which is related to the alignment, is present in the Ronchigrams. After extracting this information, it can be used in the automatic control of alignment. The process is shown schematically in Figure 1: the Ronchigram is retrieved using the image sensor; alignment information is retrieved from the image using an information extraction method; this information is then used in the control program to calculate the adjustment for the microscope settings; and if necessary the process is repeated until a quality criterion is reached.

The above equations are used to arti cially generate Ronchigrams. The object transmission function of amorphous carbon is represented by random noise [8]. Examples of simulated Ronchigrams are shown in gure 2. This gure also illustrates a newly developed information extraction method. This method can detect the shapes in Ronchigrams and derive information about the defocus and the $\mathrm{x}-\mathrm{y}$ distribution and the strength of the astigmatism. The results are shown in Figure 3a for three di erent defocus values: the radius is maximized for Scherzer defocus.

The apparent features in the Ronchigram are dependent on the structure of the sample (see Figure 3b). Due to this, contrast and apparent shape changes can occur, making information retrieval from these images a challenge, especially under near-focus conditions. Advanced averaging techniques and least-squares optimization methods (where a model is tted to the image) may be used for this purpose. A next step will be to develop a method that identi es the transfer of the microscope settings (e.g. lens, de ector and quadrupole settings) to the quantities that can be extracted from the Ronchigram (e.g. optical parameters like defocus and astigmatism). This information will then be used to design an optimal control program [9].

\section{References}

[1] Q.S. Zheng et al., J. Struct. Biol. 147 (2004) 91-101.

[2] J. Feng et al., J. Microsc. 228 (2007) 406-412.

[3] J.M. Rodenburg and E.B. Macak, Microsc. Anal. 90 (2002) 5-6.

[4] N. Dellby et al., J. Electron Microsc. 50 (2001) 177-185. 
[5] H. Sawada et al., Ultramicroscopy 108 (2008) 1467-1475.

[6] J.M. Cowley, Ultramicroscopy 4 (1979) 435-450.

[7] R.R. Meyer et al., Ultramicroscopy 99 (2004) 115-123.

[8] M. De Graef, Introduction to Conventional Transmission Electron Microscopy. Cambridge University Press, Cambridge, 2003.

[9] This research was sponsored by the Condor project at FEI company, under the responsibilities of the Embedded Systems Institute (ESI). This project is partially supported by the Dutch Ministry of Economic A airs under the BSIK program.
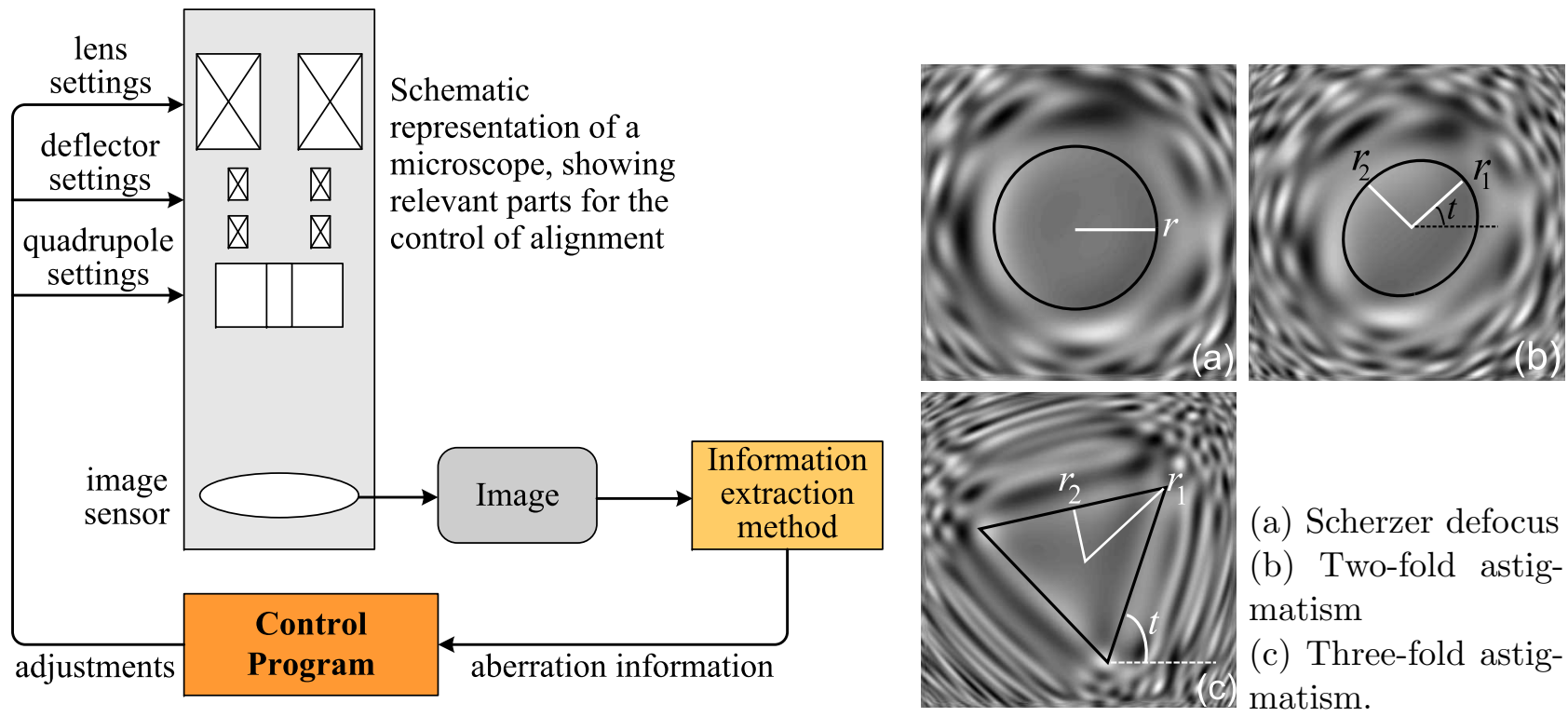

Figure 1: This diagram shows the components for alignment control. A Ronchigram image is recorded and aberration information is extracted from it. This information is then used in the control program to calculate the adjustments to the microscope settings, optimising the alignment.
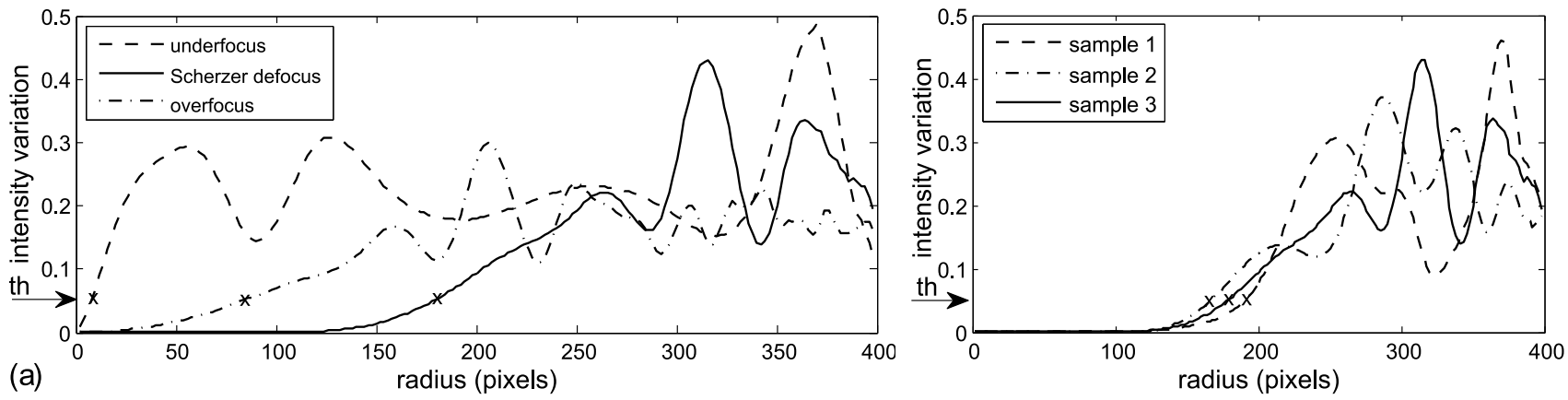

Figure 3: The intensity variation of all pixels with a certain radius from the center of the Ronchigram is shown. (a) This is plotted for three defocus values: strong underfocus; Scherzer defocus; and overfocus. The radius is found at which the variation exceeds the threshold value (th) of 0.05. This radius maximizes at Scherzer defocus and can thus be used to tune the microscope for this focus setting. (b) Intensity variations calculated for three completely di erent samples. Variations due to the sample in uence the found radius; compensation for these variations is necessary in the methods used, which is currently being investigated. 